Commun. math. Phys. 4, 64-76 (1967)

\title{
Fermions and Associated Bosons of One-dimensional Model
}

\author{
D. A. UHLENBROCK* \\ The Institute for Advanced Study, Princeton, New Jersey \\ Received July 1, 1966
}

\begin{abstract}
The representation of the canonical commutation relations involved in the construction of boson operators from fermion operators according to the recipe of the neutrino theory of light is studied. Starting from a cyclic Fockrepresentation for the massless fermions the boson operators are reduced by the spectral projectors of two charge-operators and form an infinite direct sum of cyclic Fock-representations. Kronig's identity expressing the fermion kinetic energy in terms of the boson kinetic energy and the squares of the charge operators is verified as an identity for strictly selfadjoint operators. It provides the key to the solubility of LutTingER's model. A simple sufficient condition is given for the unitary equivalence of the representations linked by the canonical transformation which diagonalizes the total Hamiltonian.
\end{abstract}

\section{Indroduction}

A number of theoretical physicists, among them DE BRoGLIE, JORDAN, BorN and NAGENDRA-NATH, have worked on a neutrino theory of light. In 1938, Pryce [1] reviewed the results of the efforts up to that time and demonstrated, that in a four-dimensional space-time version of the theory the conditions imposed by the commutation relations for the field amplitudes and the connection between spin and polarization are incompatible. Perkins [2] in his recent formal attempt avoids this difficulty but does not obtain Bose quantization for the photons.

The two-dimensional variant of the theory, which was originally developed as a proving ground for a realistic theory, has met with some interest in its own right, due to its connection with exactly soluble models. On the one hand, as stressed particularly by WIGHTMAN [3], it is closely related to the THIRRING model, while on the other hand it appears in the context of the soluble quantum mechanical many body problem due to LutTinger [4]. Mattis and Lieb [5] reconsidered this problem and considerably improved the discussion. Tomonaga [6] did some related work, which was pursued further by ENGELsBerg and VARGA.

* Work supported by the National Science Foundation. 
We wish to enhance the understanding of this model and its solution by a more refined analysis of the relation between the fermions and the associated bosons. In particular, we establish that the representation of the canonical commutation relations (CCR) afforded by the boson field is an infinite direct sum of irreducible Fock-representations in the Hilbert space of the irreducible Fock-representation of the canonical anticommutation relations (CAR) for the fermions. This reducibility results from the absence of zero-momentum boson creation and annihilation operators. More precisely, every element of the boson algebra commutes with two selfadjoint charge operators with integral spectra, which are formally obtained by the zero-momentum limit from the boson creation operators. The simultaneous spectral decomposition of these charge operators then implies the above reduction of the boson algebra. The structure of the cyclic vacua for the boson algebra, in terms of simple monomials in the fermion creation operators acting on the fermion vacuum, is the only reminder of the underlying fermion description. The special role played by momentum zero in this context is reminiscent of the infrared difficulties occurring in the two-dimensional relativistic models involving scalar fields [3], [7].

Having established the structure of the representation of the CCR it is not difficult to verify that the identity derived by KRoNig between the kinetic energy of the fermions and that of the bosons is a true operator identity for (strictly) selfadjoint operators. This identity provides the key to the solubility of the LUTTINGER model, since the interaction in question is expressible as a quadratic form in the boson operators. As a result the total Hamiltonian is essentially a quadratic form in the boson operators and can be diagonalized by a canonical transformation à la BogolIUBOv. The requirement that the canonical transformation be unitarily implementable between Fockspaces imposes certain conditions on the interaction potential. A simple sufficient condition is given. It is also seen that a local delta function interaction leads to a unitarily inequivalent representation, a fact which correlates with the puzzling results obtained by LutTinger or MATTis and Lieb for this potential.

In sections two and three we give the relevant definitions for the fermions and bosons respectively, while the reduction of the boson algebra is carried out in section four. KRONIG's identity is discussed in section five and employed in section six for the diagonalization of the total Hamiltonian.

\section{Fermions}

Imagine a one-dimensional system of two kinds of fermions in a (periodic) box of length $L$. We write $\alpha_{\tau}^{\sigma}(x)$ for the creation - (if $\sigma=+$, annihilation - if $\sigma=-$ ) operators of type $\tau$ and momentum $x$ in the

5 Commun. math. Phys., Vol, 4 
customary Fock-representation of the CAR with cyclic vacuum $\Omega^{(\alpha)} \in \mathscr{H}^{(\alpha) 1}$.

A box quantized version of a relativistic free Hamiltonian for massless fermions would have the form

$$
H_{0}^{(\alpha)}=\sum_{(\tau, x) \in S \times I^{\prime}} \tau \varkappa \alpha_{\tau}^{+}(x) \alpha_{\tau}^{-}(x)
$$

with a corresponding number operator

$$
N^{(\alpha)}=\sum_{(\tau, x) \in S \times I^{\prime}} \alpha_{\tau}^{+}(x) \alpha_{\tau}^{-}(x) .
$$

These operators are defined on the invariant dense domain $D^{(\alpha)} \equiv$ $\equiv \mathscr{P}\left\{\alpha^{+}\right\} \Omega^{(\alpha)}$ on which they are essentially selfadjoint. To avoid the negative energy contributions of (1) one employs a particle-hole canonical transformation

$$
\beta_{\tau}^{\sigma}(x) \leftrightarrow \alpha_{\tau \varepsilon(\varkappa)}^{\sigma \tau}(x), \quad(\sigma, \tau, \varkappa) \in S^{2} \times I^{\prime}
$$

where $\beta_{\tau}^{\sigma}(x)$ carries momentum $\sigma \tau \varkappa$ and energy $\sigma|x|$. Like the $\alpha$-representation the $\beta$-representation of the CAR is required to be an irreducible Fock-representation with cyclic vacuum $\Omega \equiv \Omega^{(\beta)}$, so that these two representations cannot act irreducibly in the same space ${ }^{2}$.

To the operators in Eqs. (1), ( $\left.1^{\prime}\right)$ correspond the following operators in the $\beta$-representation (the arrows emphasize the distinction of the representation spaces, which formally results in the appearance of infinite

${ }^{1}$ If $I\left(I_{+}, I_{-}, I_{0}\right)$ denotes the integers (strictly positive, strictly negative, nonzero), define $I(L) \equiv \frac{2 \pi}{L} I$ (similarly $I_{+}(L)$ etc.). Define the odd-halfinteger sets $I_{+}^{\prime} \equiv I_{+}-\frac{1}{2} \equiv\left\{j \mid j=i-\frac{1}{2}, i \in I_{+}\right\}, I_{-}^{\prime}=I_{-}+\frac{1}{2}$ and $I^{\prime}=I_{+}^{\prime} \cup I_{-}^{\prime}$ (similarly $I_{+}^{\prime}(L)$ etc.). $S$ is the set of two signs $\{+,-\} \equiv\{+1,-1\}$. Let $R$ and $C$ be the real and complex number fields. For $r \in R \quad \varepsilon(r)$ is the sign of $r$ such that $\varepsilon(0)=0$.

Under "quantization" of the free fermion field with periodic boundary conditions the wave vectors of the plane waves are restricted to be elements of $I(L)$. Except for the discussion of a limit $L \rightarrow \infty$ (in some fixed units) the dependence on $L$ is not particularly relevant in the formulae and we choose to normalize $L$ to $2 \pi$; i.e. $I(L)=I$ etc. Furthermore we resort to the convenient expedient of shifting $I$ by $\frac{1}{2}$ to get $I^{\prime}$.

Subscripts $f$ resp. $b$ and generally the use of lower case Greek resp. Latin letters distinguish fermion expressions from boson expressions.

Given a collection $\left(A_{j}\right)_{j \in J}$ of linear operators on a (complex) Hilbert space $\mathscr{H}, \mathscr{M}\left\{\left(A_{j}\right)_{j \in J}\right\}$ denotes the set of all (finite) monomials in the $A_{j}$, while $\mathscr{P}\left\{\left(A_{j}\right)_{j \in J}\right\}$ $=\mathscr{L} \mathscr{M}\left\{\left(A_{j}\right)_{j \in J}\right\}$ is the (complex) linear hull of $\mathscr{M}\left\{A_{j}\right\}$, i.e., the corresponding set of polynomials over $C$.

${ }^{2}$ For example $\Omega^{(\beta)}$ cannot lie in $\mathscr{H}^{(\alpha)}$ since it can be "approximated" in $\mathscr{H}^{(\alpha)}$ by $\Omega_{n}=\prod_{0<\tau \varkappa<n} \alpha_{\tau}^{+}(\varkappa) \Omega^{(\alpha)}$ and $\underset{n \rightarrow \infty}{w-\lim _{n}} \Omega_{n}=0$ while $\lim _{n \rightarrow \infty}\left\|\Omega_{n}\right\|=1$. 
constants)

$$
\begin{gathered}
H_{0}^{(\alpha)} \rightarrow H_{0}=\sum_{(\tau, x) \in S \times I^{\prime}}|x| \beta_{\tau}^{+}(x) \beta_{\tau}^{-}(x) \\
N^{(\alpha)} \rightarrow N=\sum_{(\tau, x) \in S \times I^{\prime}} \tau \beta_{\tau}^{+}(x) \beta_{\tau}^{-}(x) \\
\hat{N}=\sum_{(\tau, x) \in S \times I^{\prime}} \beta_{\tau}^{+}(x) \beta_{\tau}^{-}(x) .
\end{gathered}
$$

Again these operators are essentially selfadjoint on $D \equiv \mathscr{P}\left\{\beta^{+}\right\} \Omega$, where $\overline{\mathscr{P}\left\{\beta^{+}\right\} \Omega}=\mathscr{H} ; H_{0}$ is now non-negative while $N$ has lost this property of $N^{(\alpha)}$ and $\hat{N}$ therefore substitutes for $N^{(\alpha)}$. The interaction will be specified in section 6 .

\section{Associated bosons}

Heuristically the bosons are given by the Fourier components of the quantized particle density. More specifically, as shown by MATris and LIEB, the Fourier amplitudes of the particle density in the $\alpha$-representation, which form an abelian set of operators in that representation, are transformed by Eq. (2) into a set of operators (in the $\beta$-representation) which is no longer abelian but in fact constitutes a representation of the CCR.

For $(s, t, k) \in S^{2} \times I_{+}$one defines a set of (unbounded) boson operators by

$$
b^{s}(t k) \equiv b_{t}^{s}(k) \equiv k^{-\frac{1}{2}} \sum_{x \in I^{\prime}} \beta_{\varepsilon(s k+\varkappa)}^{\varepsilon(s k+\varkappa)}(t(s k+\varkappa)) \beta_{\varepsilon(x)}^{-\varepsilon(\varkappa)}(t \varkappa)
$$

with invariant domain $D$, carrying the momentum $s t k$ and energy $s k^{3}$. These operators are well defined on $D$, since the infinite sums reduce to finite ones on any element of $D$.

To clarify the structure of these operators we expand $b^{+}(k), k \in I_{+}$, as an example:

$$
\begin{aligned}
k^{\frac{1}{2}} b^{+}(k)= & \sum_{x \in I^{\prime}} \beta_{\varepsilon(k+x)}^{\varepsilon(k+x)}(k+x) \beta_{\varepsilon(x)}^{-\varepsilon(x)}(x) \\
= & \sum_{k>0} \beta_{+}^{+}(k+x) \beta_{+}^{-}(x)+ \\
& +\sum_{-k<x<0} \beta_{+}^{+}(k+x) \beta_{-}^{+}(x)+ \\
& +\sum_{x<-k} \beta_{-}^{-}(k+x) \beta_{-}^{+}(x) .
\end{aligned}
$$

The summands in the first (third) sum of Eq. (4') do not change the total

3 The connection with the notation of BorN and NAGENDRA-NATH or MATTIS and LIEB is given by

$$
\begin{gathered}
\mathrm{BN}: \beta_{\varepsilon(\varkappa)}^{-\varepsilon(\varkappa)}(\tau \varkappa)=\alpha_{\varkappa, \tau}=\left(\gamma_{-\varkappa, \tau}\right)^{+}, \\
\mathrm{ML}: b_{t}^{s}(k)=k^{-\frac{1}{2}} \varrho_{t}(s+k) .
\end{gathered}
$$


number of +-particles (--particles) but translate any occupied level by an amount $k$ away from the origin. These terms were interpreted in terms of a Raman effect for the neutrino theory of light. On the other hand the second sum is a finite sum of pair creation operators with momentum $k$, and as such does not commute with $\hat{N}$. These terms were interpreted by the rule that a "photon" of momentum $k$ is actually a superposition of neutrinos and antineutrinos of momenta $x+k$ and $x$ respectively. One verifies by direct calculation that the $b^{s}(t k)$ satisfy the CCR on D and that the (strict) adjoint $\left(\left.b_{s}(t k)\right|_{D}\right) *$ extends $\left.b^{-s}(t k)\right|_{D}$.

We will show that these boson operators in a unique way give rise to a representation of the CCR in the strict sense [8] by demonstrating that the representation involved is the restriction to $D$ of an infinite direct sum of copies of a cyclic Fock-representation.

In this connection it is essential to note that the value $k=0$ is omitted in the definition of Eq. (4). In fact, there are two operators $B_{t}$, $t \in S$, given formally as $\lim _{k \rightarrow 0} \sqrt{k}: b_{t}^{s}(k)$ :

$$
B_{t}=\sum_{x \in I^{\prime}} t \varepsilon(\varkappa) \beta_{\varepsilon(x)}^{+}(t x) \beta_{\varepsilon(x)}^{-}(t x)
$$

or

$$
\begin{aligned}
B_{t} & =N(\tau=+t, \varkappa>0)-N(\tau=-t, \varkappa<0), \\
N & =B_{+}-B_{-},
\end{aligned}
$$

so that these operators are essentially selfadjoint on $D$, where they also commute with each other as well as with the $b_{t}^{s}(k)$.

\section{Direct sum decomposition of $\mathscr{H}$}

Since the spectrum of $B_{+}$as well als $B_{-}$equals $I$, their common spectral resolution affords a direct sum decomposition of $\mathscr{H}$ of the form

$$
\mathscr{H}=\bigoplus_{\left(l, l^{\prime}\right) \in I^{2}} \mathscr{H}_{l^{\prime}, l}
$$

such that all $\mathscr{H}_{l^{\prime} l}$ are invariant subspaces for the boson algebra and one has for $\phi \in \mathscr{H}_{l^{\prime} l}$,

$$
B_{+} \phi=l \phi, \quad B_{-} \phi=l^{\prime} \phi .
$$

It remains to be shown that each $\mathscr{H}_{l^{\prime} l}$ is generated by the boson algebra applied to a cyclic vacuum. To that end we remark first that due to Eqs. (3), (4) and (5) the degrees of freedom with $\varepsilon(x) \cdot \tau=+$ for the fermions and $t=+$ for the bosons are kinematically independent from those with $\varepsilon(x) \tau=t=-.^{4}$

${ }^{4}$ More precisely, if $\mathscr{M}_{t}\left\{\beta^{+}\right\} \equiv \mathscr{M}\left\{\beta_{\tau}^{+}(x) \mid \tau \varepsilon(x)=t\right\}, \mathscr{P}_{\imath}\left\{\beta^{+}\right\}=\mathscr{L}_{\mathscr{M}_{t}}\left\{\beta^{+}\right\}$and $\mathscr{H}_{t}=\overline{\mathscr{P}_{t}\left\{\beta^{+}\right\} \Omega_{t}}$, then there is a natural isomorphism between $\mathscr{H}$ and $\mathscr{H}_{-} \otimes \mathscr{H}_{+}$ with $\Omega \leftrightarrow \Omega_{-} \otimes \Omega_{+}$such that the operators of interest split (with some abuse of notation) as $b_{+}^{s}(k) \leftrightarrow 1_{-} \otimes b_{+}^{s}(k), B_{+} \leftrightarrow 1_{-} \otimes B_{+}, b_{-}^{s}(k) \leftrightarrow b_{-}^{s}(k) \otimes 1_{+}, B_{-} \leftrightarrow B_{-} \otimes$ $\otimes 1_{+}$and $\mathscr{H}_{l^{\prime} l} \leftrightarrow \mathscr{H}_{-, l^{\prime}} \otimes \mathscr{H}_{+, l}$ etc. 
In the following we shall hence treat only the case $\varepsilon(x) \tau=+$ and $t=+$, if the alternate case can be dealt with completely analogously. Thus in the spirit of footnote 4 we deal with $b_{+}^{s}(k), B_{+}$in $\mathscr{H}_{+}=\overline{\mathscr{L} \mathscr{M}_{+}\left\{\beta^{+}\right\}}$ $=\bigoplus_{l \in I} \mathscr{H}_{+, l}$.

One observes that for any $l \in I$ the following vector

$$
\Omega_{+, l}=\underset{0<\varepsilon(l) \cdot \varkappa<|l|}{\vec{\Pi}} \beta_{\varepsilon(l)}^{+}(\varkappa) \Omega_{+}, \quad\left(\Omega_{+, 0} \equiv \Omega_{+}\right)
$$

ies in $\mathscr{H}_{+, l}$ and satisfies

$$
b_{+}^{-}(k) \Omega_{+, l}=0, \text { for all } k \in I_{+} .
$$

Again the possibility of constructing such vacua stems from the absence of zero momentum bosons: the pair annihilator part of $b_{+}^{-}(k)$ vanishes on $\Omega_{+, l}$, since there are no pairs to annihilate, while the translator part gives nothing due to the dense packing of the occupied levels between 0 and $|l|$; i.e., the particle of any occupied level will be shifted down by the +-translator into another occupied level with zero result, since double occupation is ruled out by Fermi statistics. On the other hand $B_{+}\left(\sim b_{+}^{-}(0)\right)$ does not annihilate $\Omega_{+, l}$ (except $l=0$ ).

To show that $\Omega_{+, l}$ is cyclic in $\mathscr{H}_{+, l}$ under the boson algebra it suffices to show $\mathscr{P}\left\{b_{+}^{+}\right\} \Omega_{+, l}=\mathscr{P}_{+}\left\{\beta^{+}\right\} \Omega_{+} \cap \mathscr{H}_{+, l}$, since the closure of both sides of this equation gives $\mathscr{H}_{+, l}$. Since $b_{+}^{s}(k)$ commutes with $B_{+}$and maps $D$ into $D, \mathscr{P}\left\{b_{+}^{+}\right\} \Omega_{+, l} \subset \mathscr{P}_{+}\left\{\beta^{+}\right\} \Omega_{+} \cap \mathscr{H}_{+, l}$ holds. Again in what follows the case $l<0$ is entirely analogous to the case $l>0$, so that we shall assume $l \geqq 0$ fixed.

Due to the CCR any element in $\mathscr{M}\left\{b_{+}^{+}\right\} \Omega_{+, l}$ is uniquely (up to a factor and excluding the trivial monomial) characterized by the nonincreasing sequence of the momenta $k_{1} \geqq k_{2} \geqq \cdots \geqq k_{q}>0$ of its factors $b_{+}^{+}\left(k_{i}\right)$. If $\sum_{i=1}^{q} k_{i}=K \in I_{+}$, then $\left(k_{i}\right)_{i \in I_{q}}, I_{q}=[0, q] \cap I$ is a partition of $K$ into non-negative integers and can be uniquely represented by its $\mathscr{F}$-graph:

Definition. Given $l \geqq 0, K \in I_{+}$and a partition $\left(k_{i}\right)_{i \in I_{q}}$ of $K$ into positive integers. Consider a two-dimensional cartesian coordinate system and the set of (horizontal) line segments of length $k_{1} \geqq$ $\geqq k_{2} \geqq \cdots \geqq k_{q}>0$ each. Attach the left endpoint of the longest line segment $\left(k_{1}\right)$ at the point $\left(l-\frac{1}{2}, 0\right)$, the next smaller one $\left(k_{2}\right)$ at $\left(l-\frac{3}{2}, 1\right)$ etc. Any such rooted collection of telescoped line segments will be called an $\mathscr{F}$-graph $f$ with "norm" $\|f\|=K=\sum_{i=1}^{q} k_{i}$ and "root" $l$. 
Denote the set of projections of all left (or right) endpoints of an $\mathscr{F}$-graph $f$ on the horizontal axis by $L_{f}$ (or $R_{f}$ ). The number of distinct partitions of $K$ is $p(K)$, whose generating function is $G(x) \equiv \prod_{l \geqq 1}\left(1-x^{l}\right)^{-1}$, $|x|<1$.

Due to Eq. (7) any two elements in $\mathscr{M}\left\{b_{+}^{+}\right\} \Omega_{+, l}$ with distinct $\mathscr{F}$-graphs are mutually orthogonal, in particular also those with different total momentum $K$. Hence $\mathscr{M}\left\{b_{+}^{+}\right\} \Omega_{+, l}$ can be represented as the orthogonal union over $K \in I_{+} \cap\{0\}$ of the finite subsets with $p(K)$ elements corresponding to all distinct partitions of $K$ (for $K=0$ we add the vacuum $\left.\Omega_{+, l}\right)$. Similarly $\mathscr{P}\left\{b_{+}^{+}\right\} \Omega_{+, l}=\mathscr{L} \underset{K \in I_{+} \cup\{0\}}{\cup} \mathscr{M}\left\{b_{+}^{+} \mid \sum k_{i}=K\right\} \Omega_{+, l}$.

Quite analogously, any element in $\mathscr{M}_{+}\left\{\beta^{+}\right\} \Omega_{+}$can be uniquely (up to a factor) represented by its $\mathcal{O}$-graph:

Definition. Given any element of $\mathscr{M}_{+}\left\{\beta^{+}\right\} \Omega_{+}$, its $\mathcal{O}$-graph (for occupation) is the set of the momenta $\left\{x_{j}\right\}$ occurring in the factors plotted on a (horizontal) cartesian axis; i.e. the geometrical representation by points of the corresponding subset of $I^{\prime}$.

If $\sigma$ is such a graph, let $\sigma_{+} \equiv \sigma \cap I_{+}^{\prime}, \sigma_{-} \equiv \sigma \cap I_{-}^{\prime} . \sigma$ belongs to $\mathscr{H}_{+, \imath}$ if and only if $\left|\sigma_{+}\right|=\left|\sigma_{-}\right|+l$, i.e., $\sigma_{+}$contains $l$ more elements than does $\sigma_{-}$. Set $\|\sigma\|_{l} \equiv \sum_{x \in o}|x|-\frac{1}{2} l^{2} \geqq 0$ and denote the number of distinct O-graphs o with $\|\sigma\|_{l}=K \in I_{+}$by $c_{l}(K)$. Its generating function is

with

$$
x^{-\frac{1}{2} l^{2}} \frac{1}{2 \pi} \int_{0}^{2 \pi} d \varphi e^{-i l \varphi} G(x ; \varphi)
$$

$$
G(x ; \varphi) \equiv \prod_{x \in I_{+}^{\prime}}\left(1+2 \cos \varphi x^{\varkappa}+x^{2 \varkappa}\right), \quad|x|<1 .
$$

Elements in $\mathscr{M}_{+}\left\{\beta^{+}\right\} \Omega_{+}$with distinct $\mathcal{O}$-graphs are mutually orthogonal and $\mathscr{M}_{+}\left\{\beta^{+}\right\} \Omega_{+}$is exhausted by all different $\sigma$ with $l$ running through $I$ and $\|\sigma\|_{l}$ through $I_{+} \cup\{0\}$.

Lemma. For any $K \in I_{+} \cap\{0\}$ and $l \in I$ one has $c_{l}(K)=c_{0}(K)=(8)$ $=p(K)$.

Remark. This implies a proof of the identity

$$
\prod_{l \in I_{+}}\left(1-x^{l}\right)^{-1}=x^{-\frac{1}{2} l^{2}} \frac{1}{2 \pi} \int_{0}^{2 \pi} d \varphi e^{-i l \varphi} \prod_{x \in I_{+}^{\prime}}\left(1+e^{i \varphi} x^{\varkappa}\right)\left(1+e^{-i \varphi} x^{\varkappa}\right),
$$

or in other words $x^{\frac{1}{2} l^{2}} G(x)$ is the $l$-th fourier component of $G(x ; \varphi)$ w.r.t. the $\varphi$ variable and identically in $|x|<1$.

Proof. It suffices to take $l \geqq 0$ fixed. If $\sigma$ is an $\mathcal{O}$-graph for $l>0$ with $\|\sigma\|_{l} \geqq 0$, the involutory map

$$
\sigma \rightarrow \sigma^{\prime} \equiv(\sigma-\sigma \cap[0, l]) \cup\left([0, l] \cap\left(I_{+}^{\prime}-\sigma_{+}\right)\right)
$$


(i.e., reversal of occupation in the shell $[0, l] \cap I_{+}^{\prime}$ ) maps $\sigma$ into an $\sigma^{\prime}$ corresponding to $l=0$ such that $\|\sigma\|_{l}=\left\|\sigma^{\prime}\right\|_{0}$. Thus the cardinality of the set of all $\mathcal{O}$-graphs of type $l$ with $\|\sigma\|_{l}=K$ is the same as that of the set of all $\sigma^{\prime}$ of type zero with $\left\|\sigma^{\prime}\right\|_{0}=K$ or equivalently $c_{l}(K)=c_{0}(K)$.

Next it is necessary to establish a one-to-one correspondence between the set of all $\mathscr{F}$-graphs of norm $K$ and the set of all $\mathcal{O}$-graphs of type $l=0$ and norm $K^{5}$.

Given any $\mathscr{F}$-graph $f$ the map

$$
f \rightarrow o(f) \equiv R_{f} \cup L_{f}-R_{f} \cap L_{f}
$$

gives an $\mathcal{O}$-graph, since for any element in $R_{f}$ there is at most one element in $L_{f}$ to which it is equal, owing to the decreasing property of the partition parts and the telescoped form of $f$. Conversely, given any $\mathcal{O}$-graph $\sigma$, let $q=\frac{1}{2}-\min \{x \mid x \in \sigma\}$ and define $L(\sigma)=[-q, 0] \cap I^{\prime}, R(\sigma)=\sigma_{+} \cup$ $\cup\left(L(\sigma)-\sigma_{-}\right)$, both ordered as subsets of $I^{\prime}$. Then $\sigma \rightarrow L(\sigma) \cup R(\sigma)$ yields a telescoped set of pairs (of endpoints of line segments) if one pairs the largest elements in $L(\sigma)$ with the largest one in $R(\sigma)$, the next smaller with the next smaller etc. One checks easily that the two maps are injective and mutually inverse, so that $p(K)=c_{0}(K)$. The identity then follows by comparing the generating functions for $p(K), c_{0}(K)$ and $c_{l}(k)$ respectively. Q.E.D.

This lemma implies that for any fixed $l$ the number of linearly independent elements $\sigma$ in $\mathscr{P}_{+}\left\{\beta^{+}\right\} \Omega_{+} \cap \mathscr{H}_{+, l}$ with $\|\sigma\|_{l}=K \geqq 0$ is equal to the number of linearly independent elements $f$ in $\mathscr{P}\left\{b_{+}^{+}\right\} \Omega_{+, \imath}$ with $\|f\|=K$. Therefore one finally concludes that

$$
\begin{aligned}
\mathscr{P}\left\{b_{+}^{+}\right\} \Omega_{+, l}=\mathscr{L} \underset{K \geqq 0}{\bigcup}\{f \mid\|f\| & =K\} \\
& =\mathscr{L} \bigcup_{K \geqq 0}\left\{\sigma \mid\|\sigma\|_{l}=K\right\}=\mathscr{P}_{+}\left\{\beta^{+}\right\} \Omega_{+} \cap \mathscr{H}_{+, l},
\end{aligned}
$$

or $\mathscr{H}_{+, l}=\overline{\mathscr{P}\{+\} \Omega_{+, l}}$. Recalling some of the well-known properties of the Fock-representation [9] we arrive at the

Proposition. Let $\beta_{\tau}^{\sigma}(\varkappa),(\sigma, \tau, \varkappa) \in S^{2} \times I^{\prime}$ be a Fock-representation of the CAR with cyclic vacuum $\Omega$ in the Hilbert space $\mathscr{H}=\mathscr{P}\left\{\beta_{\tau}^{+}\right\} \Omega$; let the boson operators $b_{t}^{s}(k),(s, t, k) \in S^{2} \times I_{+}$and $B_{t}, t \in S$ be defined by Eqs. (4) and (5) on $D=\mathscr{P}\left\{\beta_{\tau}^{+}\right\} \Omega$, then the decomposition of $\mathscr{H}$ in Eq. (6) by the spectral projectors of the essentially selfadjoint $B_{t}$ induces a direct sum decomposition of the reducible boson algebra into irreducible cyclic components, each of which gives rise to a Fockrepresentation of the CCR.

5 I owe the geometric idea of this map to E. NeLson. 
The unique cyclic vacuum in $\mathscr{H}_{l^{\prime}, l}$ is expressed as

$$
\Omega_{l^{\prime}, l}=\prod_{\substack{0<\varepsilon\left(l^{\prime}\right) x^{\prime}<\left|l^{\prime}\right| \\ 0<\varepsilon(l) x<|l|}} \beta_{-\varepsilon\left(l^{\prime}\right)}^{+}\left(x^{\prime}\right) \beta_{\varepsilon(l)}^{+}(x) \Omega, \quad\left(\Omega_{0,0} \equiv \Omega\right) .
$$

Also the operators

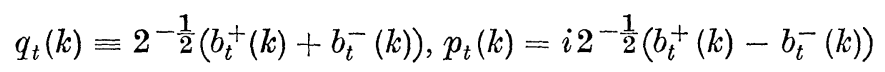

are essentially selfadjoint on $D$ and after exponentiation give rise to a strongly continuous representation of the CCR in the Weyl form, which acts irreducibly in each subspace $\mathscr{H}_{l^{\prime}, l}$.

Remark. Let $\beta_{l^{\prime}, l}$ denote the $\beta$-monomial in Eq. (9) (i.e., $\Omega_{l^{\prime}, l}$ $\left.=\beta_{l^{\prime}, l} \Omega_{00}\right)$ and define projectors $\nu_{l^{\prime}, l}$ and $\bar{\nu}_{l^{\prime}, l}$ by $\nu_{l^{\prime}, l} \equiv\left(\beta_{l^{\prime} l}\right)^{+} \beta_{l^{\prime} l}$ and $\bar{v}_{l^{\prime}, l} \equiv \beta_{l^{\prime} l}\left(\beta_{l^{\prime} l}\right)^{+}$. Then $\beta_{l^{\prime} l}$ induces a bounded linear map $f_{l^{\prime} l}: \mathscr{H}_{00} \rightarrow$ $\rightarrow \mathscr{H}_{l^{\prime} l}$ by $f_{l^{\prime} l}(\phi)=\beta_{l^{\prime} l} \phi \in \mathscr{H}_{l^{\prime} l}$ for $\phi \in \mathscr{H}_{00} \cdot f_{l^{\prime} l}$ clearly has the canonical decomposition [10]

$$
f_{l^{\prime} l}: \mathscr{H}_{00} \rightarrow \operatorname{Coim} f_{l^{\prime} l}=\bar{\nu}_{l^{\prime} l} \mathscr{H}_{00} \stackrel{\beta_{l^{\prime} l}}{\longrightarrow} \operatorname{Im} f_{l^{\prime} l}=\nu_{l^{\prime} l} \mathscr{H}_{l^{\prime} l} \rightarrow \mathscr{H}_{l^{\prime} l^{\prime}}
$$

Similarly the adjoint map $\left(f_{l^{\prime} l}\right)^{+}$is induced by $\left(\beta_{l^{\prime} l}\right)^{+}$and the inverse chain of Eq. $\left(9^{\prime}\right)$. Thus $\beta_{l^{\prime} l}\left(\right.$ or $\left.\left(\beta_{l^{\prime} l^{+}}\right)\right)$is the zero map on $\left(1-\bar{\nu}_{l^{\prime} l}\right) \mathscr{H}_{\mathbf{0 0}}$ (or $\left(1-v_{l^{\prime} l}\right) \mathscr{H}_{l^{\prime} l}$ ) and is isometric from $\bar{\nu}_{l^{\prime} l} \mathscr{H}_{00}$ (or $v_{l^{\prime} l} \mathscr{H}_{l^{\prime} l}$ ) onto $v_{l^{\prime} l} \mathscr{H}_{l^{\prime} l}\left(\right.$ or $\left.\bar{v}_{l^{\prime} l} \mathscr{H}_{00}\right)$.

\section{Identity for the fermion and boson kinetic energies}

The analysis of section 4 permits us to conclude that the boson kinetic energy

as well as the operator

$$
H_{0}^{(b)} \equiv \sum_{(t, k) \in S \times I_{+}} k b_{t}^{+}(k) b_{t}^{-}(k)
$$

$$
\frac{1}{2}\left(B_{+}^{2}+B_{-}^{2}\right)+H_{0}^{(b)}
$$

are essentially selfadjoint on $D$. By adapting the notation to that of BorN and NAGENDRA-NATH [1] one verifies that an application of their algebraic arguments for the cases $t= \pm$ separately gives the identity (due to Kronig)

$$
H_{0}^{(f)}=H_{0}^{(b)}+\frac{1}{2}\left(B_{+}^{2}+B_{-}^{2}\right) \text { on } D .
$$

Since both sides of Eq. (11) are essentially selfadjoint on $D$, their (strictly) selfadjoint closures coincide also and (11) extends as a true operator identity to the domain of these closures. On the face of it this identity is quite surprising inasmuch as the l.h.s. involves only an infinite 
sum of products of two fermion operators, while the operators appearing on the r.h.s. can be written in terms of summands with four fermion operators each. The subtle cancellation which results in Eq. (11) is, of course, due to the CAR, and the vanishing mass of the fermions.

\section{Interacting fermions and Bogoliubov transformation}

The identity (11) is the key to the solubility of the Luttinger model as well as the feasibility of the neutrino theory of light (in one dimension).

The interaction Hamiltonian of the LUTTINGER model in the notation of MATTIS and LIEB takes the form (double dots indicate normal ordering)

$$
H_{1}=\frac{1}{2} \sum_{\left(t, t^{\prime}, k\right) \in S^{2} \times I_{+}} v(k): \varrho_{t}(k) \varrho_{t^{\prime}}(k):, \quad \text { with the }
$$

interaction potential $v(0)=0, v(k)=v(-k)=v^{*}(k)$. In the $\beta$-representation $H_{1}$ commutes with $N=B_{+}+B_{-}$but not with $\hat{N}$; i.e. $H_{1}$ does not conserve the number of $\beta$-fermions. This is immediately obvious if Eq. (12) is written in terms of boson operators:

$$
H_{1}=\frac{1}{2} \sum_{k \in I_{+}} k v(k):\left[b^{+}(k)+b^{-}(-k), b^{+}(-k)+b^{-}(k)\right]_{+}:,
$$

so that with the aid of Eqs. (10), (11) and (12') the total Hamiltonian can be conveniently written as

$$
\begin{aligned}
H \equiv & H_{0}+H_{1}=\frac{1}{2}\left(B_{+}^{2}+B_{-}^{2}\right)+\sum_{k \in I_{+}}\left\{k ( 1 + v ( k ) ) \left(b^{+}(k) b^{-}(k)+\right.\right. \\
& \left.\left.+b^{+}(-k) b^{-}(-k)\right)+k \cdot v(k)\left(b^{+}(k) b^{+}(-k)+b^{-}(k) b^{-}(-k)\right)\right\} .
\end{aligned}
$$

It is easily derived that $\Omega_{l^{\prime}, l}$ is in the domain of $H$ if and only if

$$
\sum_{k \in I_{+}} k^{2} v(k)^{2}<\infty
$$

If $v(k)$ is restricted such that

$$
v(k) \geqq-\frac{1}{2}+\varepsilon, \quad \varepsilon>0, \text { all } k \in I_{+},
$$

then each summand in the sum of Eq. (13) can be diagonalized by means of a suitable Bogoliubov transformation [11]:

with

$$
\begin{aligned}
b^{s}(k) \rightarrow c^{s}(k) & \equiv U(\chi(k)) b^{s}(k) U^{+}(\chi(k)) \\
& \equiv \cosh [\chi(k)] \cdot b^{s}(k)+\sinh [\chi(k)] \cdot b^{-s}(-k)
\end{aligned}
$$

$$
\chi(k)=\chi(-k)=\overline{\chi(k)}=\tanh ^{-1}\left\{\frac{1+v(k)-\sqrt{1+2 v(k)}}{v(k)}\right\}
$$

and

$$
U(\chi(k))=\exp \left\{\chi(k)\left(b^{-}(k) b^{-}(-k)-b^{+}(k) b^{+}(-k)\right)\right\}
$$


A term in Eq. (13) is then transformed into the form

$$
\begin{aligned}
& k \cdot \sqrt{1+2 v(k)} \cdot\left(b^{+}(k) b^{-}(k)+b^{+}(-k) b^{-}(-k)\right)+c(k), \\
& \text { with } \quad c(k)=k \cdot(\sqrt{1+2 v(k)}-1-v(k)) .
\end{aligned}
$$

For arbitrary $v(k)$ the product $\prod_{k>0}^{N} U(\chi(k))$ does not necessarily converge to a unitary operator as $N \rightarrow \infty$ [12]. To find a suitable restriction on the $v(k)$ which insures the unitary implementability of the canonical transformation for all $k$ simultaneously, it is useful to recall that the Fockrepresentation of the $b^{s}(k)$ can be constructed in the form of an incomplete infinite tensor product of the representations for the various $k$-values with the reference vector $\bigotimes_{k \in I_{0}} \Omega_{b(k)}$. (Here $\Omega_{b(k)}$ corresponds to the cyclic vector for the representation of $b(k))$. $c^{s}(k)$ has the reference vector $\bigotimes \Omega_{c(k)}$. If $U(\chi(k))$ implements $b^{s}(k) \rightarrow c^{s}(k)$ then $U(\chi(k)) \Omega_{b(k)}$ $k \in I_{0}$ $=\exp (i \delta(k)) \cdot \Omega_{c(k)}$ with some real phase $\delta(k)$.

Now according to a theorem of Klauder, McKenna and Woods [8] the $b$-representation is unitarily equivalent to the c-representation if and only if $\bigotimes_{k \in I_{0}} \Omega_{b(k)}$ is weakly equivalent to $\bigotimes_{k \in I_{0}} \Omega_{c(k)}$, i.e. if and only if

$$
\sum_{k \neq 0}||\left(\Omega_{b(k)}, \exp (-i \delta(k)) U(\chi(k)) \Omega_{b(k)}\right)|-1|<\infty .
$$

Since $\left(\Omega_{b(k)}, U(\chi(k)) \Omega_{b(k)}\right)=[\cosh \chi(k)]^{-1}$ the condition (17) amounts to

$$
\sum_{k>0}(1-\operatorname{sech} \chi(k))<\infty
$$

which in turn is equivalent to

$$
\sum_{k>0} \chi(k)^{2}<\infty^{6}
$$

It follows easily, if condition (14') is obeyed and $\Omega_{00}$ is in the domain of $H$ (or equivalently condition (14) holds), that Eq. $\left(17^{\prime \prime}\right)$ applies also and $|C|<\infty$, where

$$
C \equiv \sum_{k \in I_{+}} k[\sqrt{1+2 v(k)}-1-v(k)] .
$$

6 I am grateful to O. E. LANFORD III for calling my attention to the above theorem and for showing me his similar derivation of Eq. $\left(17^{\prime \prime}\right)$. The truth of the equivalence of $\left(17^{\prime}\right)$ and $\left(17^{\prime \prime}\right)$ follows, since $1-\operatorname{sech} \chi(k) \geqq 1-\left(1+\chi(k)^{2}\right)^{-1} \geqq$ $\geqq \chi(k)^{2}$ always, and $1-\operatorname{sech} \chi(k) \leqq 1-\left(1+\frac{\chi(k)^{2}}{2} \cosh \chi(k)\right)^{-1}<\frac{1}{2} \cosh \chi(k) \times$ $\times \chi(k)^{2} \leqq$ const $\chi(k)^{2}$, if $\cosh \chi(k)$ is bounded. Thus $\left(17^{\prime}\right)$ implies $\left(17^{\prime \prime}\right)$ directly and conversely $\left(17^{\prime \prime}\right)$ implies $\lim \chi(k)=0$ or sup $\cosh \chi(k)<\infty$ and hence also $\left(17^{\prime}\right)$. 
These conditions are hence sufficient for the Hamiltonian $H$ of Eq. (13) to be unitarily equivalent to

$$
\begin{aligned}
\tilde{H}=C & +\frac{1}{2}\left(B_{+}^{2}+B_{-}^{2}\right)+ \\
& +\sum_{k>0} k \cdot \sqrt{1+2 v(k)}\left\{b^{+}(k) b^{-}(k)+b^{+}(-k) b^{-}(-k)\right\} .
\end{aligned}
$$

This solves the diagonalization problem. In each irreducible (boson) subspace $\mathscr{H}_{l^{\prime}, l}$ the groundstate energy $\frac{1}{2}\left(l^{2}+l^{\prime 2}\right)$ is shifted by the constant $C$ of Eq. (18) and the energy spectrum is modified by the factor $\sqrt{1+2 v(k)}$. Condition $\left(14^{\prime}\right)$ is seen to insure that this factor stays realvalued and bounded away from zero. The trivial structure of the excitations in the diagonalized form becomes quite complicated, when one returns to the nondiagonal form by means of the canonical transformation.

\section{Conclusion}

The above model can be modified to include an electron-phonon interaction of the usual form without losing the property of being diagonalizable by a simple (linear) canonical transformation [5], [6].

LUTTINGER was especially interested in studying the behavior of the occupation probability $n_{k}$ as a function of the momentum $k$ in the vicinity of the Fermi momentum $k_{F}$. He concluded that the behavior is characterised by a parameter $\alpha \equiv \frac{\lambda}{4 \pi^{2}} v\left(0_{+}\right)^{2}$, where $\lambda=$ interaction strength parameter, $v\left(0_{+}\right)=\lim _{k \rightarrow 0} v(k)$. MATTIS and LiEB confirmed his discussion, except for the change $v\left(0_{+}\right)^{2} \rightarrow u(0)^{2} \equiv\left[1-\left(\frac{\lambda v\left(0_{+}\right)}{\pi}\right)^{2}\right]^{-1 / 2}-1$. For $\alpha=0$ the Fermi surface has the usual stepfunction character known from the non-interacting case. If, on the contrary, $0<2 \alpha<1$ (or $1<2 \alpha$ ) then $n_{k}$ is found to be continuous at $k_{F}$ with infinite (or finite) slope. Perturbation theory seems to be consistent with the case $0<2 \alpha<$ $<1$. The infinite volume limit $L \rightarrow \infty$ is critical here.

Acknowledgement. The author is grateful to Drs. O. E. Lanford, A. C. Licht and H. ReeH and Professors E. H. LieB, E. Nelson and A. S. Wightman for remarks and conversations. He thanks Professors J. M. LÉvy and F. LURÇAT for an interesting stay in Cargèse, Corsica, during the summer of 1965 and Professor OpPenHeImer for his fine hospitality at The Institute for Advanced Study.

\section{References}

[1] Pryce, M. H. L.: Proc. Roy. Soc. (London), Ser. A 165, 247 (1938). This article also reviews the earlier work and the relevant references are to be found there. Among these the following are particularly relevant to our discussion: Born, M., and N. Nagendra-Nath: Proc. Ind. Acad. Sci. 3, 318 (1936), N. NAGENDRA-NATH, ibid. p. 448. 
[2] Perkins, W. A.: Phys. Rev. 137, B1291 (1965).

[3] Wightman, A. S.: Introduction to some aspects of the relativistic dynamics of quantical fields, I.H.E.S. Bures-sur-Yvette, S.-O., France, May 1964.

[4] Luttinger, J. M.: J. Math. Phys. 4, 1154 (1963).

[5] Mattis, D. C., and E. H. Lieb: J. Math. Phys. 6, 304 (1965).

[6] Tomonaga, S.: Progr. Theoret. Phys. (Kyoto) 5, 544 (1950). - Engelsberg, S., and B. B. VARga: Phys. Rev. 136, A 1582 (1964).

[7] Schroer, B.: Fortschr. Physik 11, 1 (1963).

[8] For example: Araki, H.: J. Math. Phys. 1, 492 (1960). - J. R. KlaUder, and J. MoKenna: J. Math. Phys. 6, 68 (1965). Also Klauder, J. R., J. McKenna, and E. J. Woods: J. Math. Phys. 7, 822 (1966).

[9] Cook, J. M.: Trans. Am. Math. Soc. 74, 222 (1953). - Araki, H., and E. J. Woods: J. Math. Phys. 4, 637 (1963). - Araki, H., and W. Wyss: Helv. Phys. Acta 37, 136 (1964).

[10] Bourbakr, N.: Eléments de mathématique. Algèbre, Ch. 2, p. 18; Paris: Hermann 1964.

[11] Bogoliubov, N. N.: J. Phys. (U.S.S.R.) 11, 23 (1947). - Ezawa, H.: J. Math. Phys. 6, 380 (1965).

[12] Segal, I. E.: Math. problems of Rel. Physics. Am. Math. Soc. 1963. 